\title{
Teaching Reform and Innovation of Embedded System Development in the Context of Artificial Intelligence
}

\section{Chen Pan}

School of International Cooperation and Exchange, Jinan Engineering Vocational Technical College, Jinan, Shandong, China

\begin{abstract}
Embedded system is a special computer application system embedded in the object system. It is a combination of software and hardware. After decades of development, embedded systems have gradually entered many important industries such as industrial production, military, aerospace, transportation, wireless communications, and consumer electronics products, and their applications are extensive. The diversity of embedded systems has driven the rapid development of the embedded product market. In this paper, we analyze the teaching reform of embedded system development from the perspective of artificial intelligence. The sample models are integrated to help understand the proposed methodology. The numerical analysis proves the novelty.
\end{abstract}

Keywords: artificial intelligence background; embedded systems; Internet of Things; system framework; hierarchical systems

\section{Introduction}

The world today is entering a period of new economic development dominated by the information industry. Information technology, as the leading technology, has penetrated into various fields of economic and social life. In the new round of scientific and technological revolutions and industrial changes in the world, the Internet + traditional industries have achieved cross-border integration and development in various fields, with broad prospects and unlimited potential. The embedded system is an important part of the Internet of Things. There is a strong demand for embedded technology talents in society and there is a broad market for learning embedded technology.

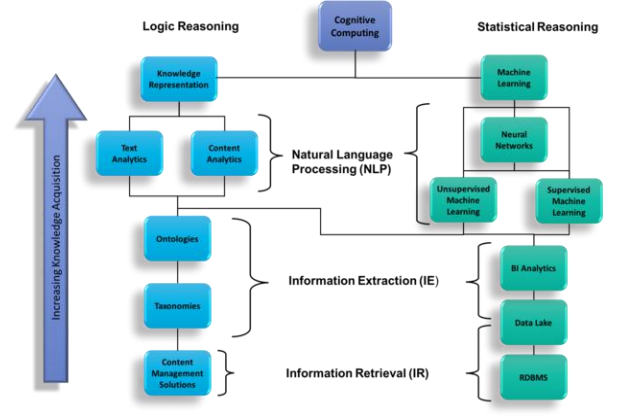

Figure 1. The systematic framework of the AI

Copyright $(2020$ by author(s) and Frontier Scientific Research Publishing Inc.

This work is licensed under the Creative Commons Attribution International License (CC BY 4.0).

http://creativecommons.org/licenses/by/4.0/ 
The embedded system is an application-centric, computer-based, and hardware-software-customizable specialpurpose computer system that meets the strict requirements for function, reliability, cost, volume, and power consumption. It is a complex of software and hardware. In recent years, the embedded product market has been booming, and embedded systems have infiltrated into industrial production, military defense, and everyday life, and have a wide range of applications. With the advent of the Internet of Things, the society has an urgent need for embedded technology professionals, and the application of embedded technology has a very broad prospect.

Embedded system applications are an important branch of computers, involving many disciplines such as computer science, electronic technology, and communications. The embedded system development course has many teaching contents. The hardware includes ARM processor architecture, hardware structure, hardware circuit and interface technology; the software includes instruction system, assembly language programming, C programming, embedded operating system and development. Students studying embedded system development courses are required to have advanced courses such as: circuit analysis basics, analog/digital electronics, microcomputer principles and applications, computer composition principles, $\mathrm{C}$ language programming, assembly language, operating system and other courses. In the organization of the curriculum system, students are required to be familiar with the hardware architecture of the embedded system, the composition and use of the hardware development platform supporting the ARM processor, master the development and debugging process of the embedded Linux application, and be able to build the embedded development environment. Compile and port the embedded Linux system; enable students to have the initial ability to independently develop a more complete embedded system. The project approach as a practical approach, in recent years, suffered from the vast number of teachers and students alike. This method is for the project to lead the students in the teacher's guide the independent complete a project that will apply what they have learned in the classroom to the project, and in a timely manner, the theory into practice. For the embedded system development course, we can consider the project based teaching and the MOOC based teaching, and in the following content, we introduce each, respectively.

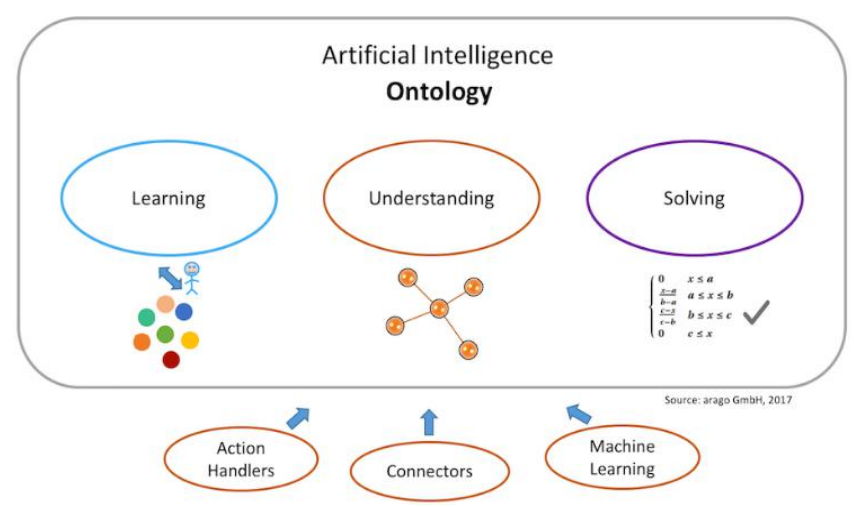

Figure 2. The AI pipelines and structures

Embedded system development and other related courses is a collection of many theories and practices in the integration of a comprehensive application classes, in the actual application in the field of practice more important than theory. For the non-focus of the undergraduate colleges in terms of trying to get our students in the community have a place and then the culture of the application type, skills type of the talent is particularly important. For embedded courses, not only to pay attention to theoretical teaching, more emphasis on practice teaching, so that students can also have the skills to be able to faster and better integrate into society. Therefore, it should increase the experimental teaching efforts, such as the appropriate increase in the experimental class and the like. In order to ensure the smooth implementation of project teaching method in teaching practice, should be regularly embedded laboratory open to all the students, teachers can come to when open laboratories, and also the students discuss issues, understand the student's master degree, in order 
to better arrange the back of the teaching content and teaching progress. Therefore, we should consider 2 major aspects. (1) At the beginning of the course, 1 2 confirmatory experiments are arranged to enable students to understand and familiarity with the process of embedded development first. In the process of verification, it is not entirely copy of the existing things, let the students modify some of the small functions, from the color, size and other simple modification of the page, to the code function modification. (2) In the process of the verification + modification, students have a general understanding of embedded development. Next, the project teaching method is applied throughout the design practice process. According to the curriculum needs to select one or several items of moderate difficulty, the project will be handed over to the students in groups or independently, from the initial collection of information to the design plan, from the implementation of the project to the final implementation, all by themselves to complete.

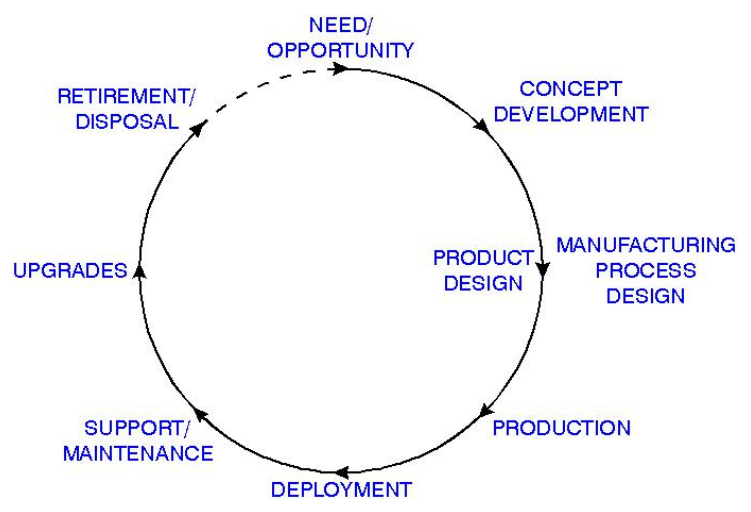

Figure 3. The project teaching circle

MOOC is an abbreviation for the Massive Open Online Course. The platform is characterized by the openness, wide range, and convenience. This model is a recent popular new course format. For the integration, we can consider the following 2 major aspects for the references. (1) The teaching video data of the course of the lesson, the entire teaching video of the course of the course selection screen photography, that is, select the screen video program, recording teaching courseware, program and experimental part of the explanation module to complete the production of teaching video. Then the teaching video is uploaded to the website of the potato website and Youku website, while the learners use the network mode to learn. The editor of the teaching video of the whole course uses all parts of the teaching material as the center, and the basic part of the courseware is the unit, and the learners view it directly from the computer, so that the image is clear and the data of each part are large, and the screen pen, handwriting plate and the camera device are selected to perfect. (2) MOOC completion of the program and curriculum design, embedded operating course is characterized by a strong curriculum and practical hands-on lab to assist students with solid knowledge and learning knowledge into valuable operational capacity. To improve the teaching system, each chapter contains the lab materials, and in the technical forum is given in the lab, and the lab program that contains two parts.

\section{The Teaching Reform and Innovation of Embedded System Development in the Context of} Artificial Intelligence

\subsection{Artificial intelligence}

The research of artificial intelligence is highly technical and professional. Each branch is in-depth and unrelated, and thus involves a very wide range. The main contents of the research on artificial intelligence include: knowledge representation, automatic reasoning and search methods, machine learning and knowledge acquisition, knowledge processing systems, natural language understanding, computer vision, intelligent robots, and automatic program design.

Knowledge representation is one of the basic problems of artificial intelligence. Inference and search are closely related to the representation method. The commonly used knowledge representation methods are: logical representation, 
production representation, semantic network representation, and frame representation.

Automatic reasoning in problem solving is the process of using knowledge. Because there are multiple methods of knowledge representation, there are various reasoning methods. The reasoning process can generally be divided into deductive reasoning and non-deductive reasoning. Predicate logic is the basis of deductive reasoning. The inheritance performance reasoning under structured representation is non-deductive. Due to the need of knowledge processing, many inference methods have been proposed in recent years, such as connection mechanism reasoning, analog reasoning, example-based reasoning, anti-inference reasoning, and limited reasoning.

Search is a problem solving method of artificial intelligence. The search strategy determines the priority relationship of knowledge used in an inference step of problem solving. It can be divided into blind search without information guidance and heuristic search guided with experience knowledge. Heuristic knowledge is often represented by a heuristic function. The more fully heuristic knowledge is used, the smaller the search space for problem solving. Typical heuristic search methods include $\mathrm{A}^{*}$ and $\mathrm{AO}^{*}$ algorithms. In recent years, research on search methods has begun to pay attention to hyperscale search problems with millions of nodes.

Machine learning is another important topic of artificial intelligence. Machine learning refers to the process of acquiring new knowledge in the sense of a certain knowledge representation. According to the different learning mechanisms, there are inductive learning, analytical learning, connection mechanism learning, and genetic learning.

The knowledge processing system is mainly composed of a knowledge base and an inference engine. The knowledge required by the knowledge base storage system, when the amount of knowledge is large and there are multiple representation methods, the rational organization and management of knowledge is important. In the problem solving, the inference engine specifies the basic methods and strategies for using knowledge. In the inference process, a database or blackboard mechanism is required for recording results or communication. If knowledge stored in the knowledge base is expert knowledge in a field (such as medical diagnosis), such a knowledge system is called an expert system. In order to meet the needs of solving complex problems, a single expert system develops to a multi-agent distributed artificial intelligence system. At this time, knowledge sharing, inter-subject cooperation, and emergence of contradictions will be the key issues for research.

\subsection{Embedded system development course}

The embedded system has the features of embedded, built-in computer, and specificity, and it depends on constantly improving basic technologies and application technologies in various fields. The extensive technical field involved in the embedded system development process is the intersection and integration of disciplines such as computer science, electronic technology, microelectronics, and automatic control. Therefore, the teaching content of the embedded system development course involves many aspects. The hardware includes ARM processor architecture, hardware structure, hardware circuit and interface technology. The software includes instruction systems, program design, assembly programming, C language programming, and embedded operating systems. The curriculum includes hardware, software knowledge and its comprehensive applications, and the comprehensiveness is very strong.

The embedded system development curriculum is highly practical. Experiments are the key to students mastering embedded system design techniques. Through experiments, students can help to consolidate relevant theoretical knowledge learned in the classroom, increase perceptual knowledge, inspire students' interest in learning, and help students correctly understand embedded system development and design processes. It helps students understand the basic system and structure of embedded system hardware, and is familiar with the operating mode of the system, and fully grasps the methods of designing, developing, and debugging embedded systems. 
As the embedded system curriculum has more teaching knowledge, the hardware and software resources involved are also more. In the selection of modern teaching materials, the theoretical knowledge system should be selected as systematically and continuously as possible. The experimental content is intuitive and novel, and it can be closely related to the actual teaching materials. Combining with the current development status of embedded technologies, in order to meet the market's demand for embedded talents and we selected ARM hardware platforms and embedded Linux operating systems as two aspects of teaching content, and explained Realview MDK as the main development environment. In the teaching process, teachers integrate some simple and practical knowledge in embedded scientific research into teaching, thus enriching the teaching content. Under this condition, we should then meet with the following challenges.

2.2.1Embedded teaching is not simply to set up several related courses, it is also called the embedded learning. Indoctrination teaching methods will not develop students who adapt to the society, but will only make students more and more rigid and will not innovate. Teachers will not only put knowledge into practice. Teaching to students, but also to cultivate the ability of independent innovation and the ability to learn in the future, no teacher can give all the knowledge to students.

2.2.2Embedded people optimistic about the prospects for many copycat opened embedded teaching in university, but teachers short of, course basically by computer science and electrical and mechanical aspects of the teacher, teachers have little practical experience, more talk about any project experience, therefore in the teaching can be carried out in accordance with the books' contents, the teaching way even lessons again also could not develop in line with market needs, we are afraid fully embedded talent.

2.2.3Embedded learning requires a lot of the experimental equipment, a lot of colleges and universities because of financial reasons, many must be the experiments the equipment is not in place, resulting in students cannot be normal embedded research, many students in the end did not complete did an experiment. Even if there are some colleges and universities to improve the corresponding experimental conditions, but because students are afraid during the experiments in the damage to the element that still not able to bold exercises.

The embedded system development course is a comprehensive course combining systematism and software and hardware. It is divided into hardware development direction and software development direction. Both directions require students to have basic capabilities in the development, design, commissioning and maintenance of embedded systems. The software programming of students in computer science and technology is a specialty, so it is more inclined to embedded software development, focusing on learning assembly language programming, data structures, embedded system principles, computer networks, Linux operating systems and other related knowledge, focusing on Programming application.

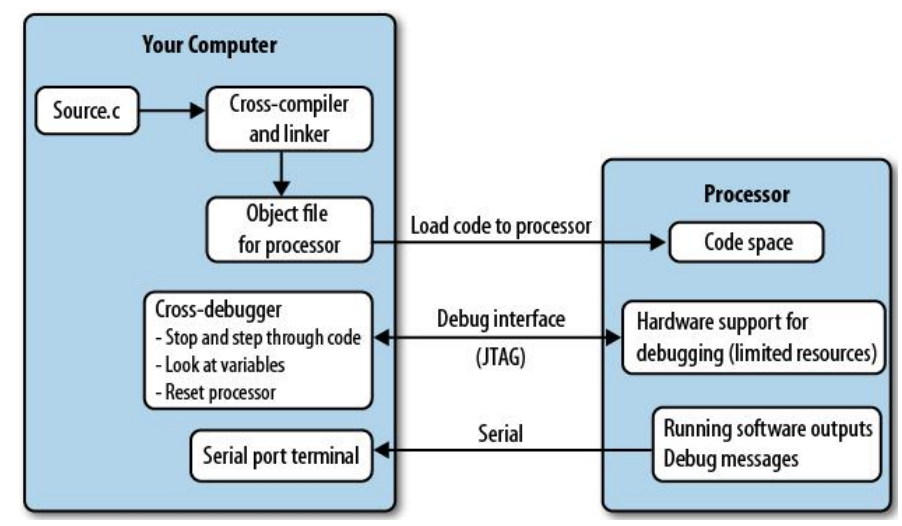

Figure 4. The hardware communication as the sample 


\subsection{Teaching reform of embedded system development practice course}

In teaching, the enthusiasm for teaching and learning is fully utilized. Teachers raise questions and through inspiration and guidance, students discuss issues in groups, freely express their understanding of issues, and seek ways and means to solve problems. This open and exploratory teaching method can mobilize students' enthusiasm for learning, exercise their thinking, improve their ability to analyze and solve problems, and cultivate students' teamwork spirit. It is conducive to inspiring and cultivating students' ability to develop innovation and has obvious advantages in cultivating applied talents.

In the teaching, full consideration is given to the cultivation of applied talents and to cultivate students' innovative spirit and innovation ability. During the teaching process, driven by actual projects, the teachers' research projects, undergraduate innovation projects, and various design competition propositions were dissected and simplified, and they were refined into individual teaching examples, so that teaching was closely related to reality. Each class teacher actively participates in the students' extracurricular embedded product production and electronic competition training activities, and encourages students to deeply understand the problems encountered in the course study through practical production, and has played a very good role in the promotion of education reform.

At present, the practical teaching of embedded system development is composed of several parts: confirmatory experiment, comprehensive design experiment, curriculum design, scientific research project, and scientific and technological innovation. The verification experiment of embedded system development is the basis of all kinds of practical teaching. The main contents of the experiment are closely related to the teaching content of the theoretical course and it is the focus of experimental teaching. The comprehensive design experiment is an extension of the confirmatory experiment. Students are further trained to apply the learned knowledge comprehensively to develop and design an embedded system. Embedded curriculum design is an important part of curriculum practice. The curriculum design is a good synthesis of what the curriculum has learned. Through this practice, it has achieved the goal of cultivating a high level of embedded developers. Scientific research project is one of the characteristics of embedded system development practice teaching. It combines today's embedded application in frontier science and technology and sets up a project suitable for practical teaching. It can broaden students' horizons and ideas, and it is good for teaching. Supporting role. Science and technology innovation is a team that students set up spontaneously to make use of after-school hours to conduct experiments, make full use of the resources of open labs, and conduct innovative experiments under the guidance of teachers. Through participation in science and technology innovation, students have accumulated rich experience in project development and cultivated their own innovation capabilities, laying a solid foundation for further embedded project R\&D.

Through the above-mentioned practice teaching, students' enthusiasm, initiative and creativity are fully mobilized. Students benefited from the practical teaching process, greatly improved the ability to independently develop and design embedded systems, and achieved good teaching results.

Make full use of modern network resources to build a platform for interaction between teachers and students. Combining traditional classroom questioning, answering questions, and discussion methods with the Internet, we use a variety of communication methods to provide students with more scientific and convenient guidance, answer questions, and promote student learning.

Based on the IoT-driven embedded system teaching reform and practice, the main purpose is to enable students to learn the basic development process of the embedded system in a simulated real development environment. Based on this, the practice innovative projects. 


\section{Conclusions}

Through the above-mentioned series of curriculum teaching reforms, students' enthusiasm, initiative and creativity have been effectively mobilized, and students' ability to analyze problems and think independently has been improved, and good teaching results have been achieved. Practical teaching has accumulated rich experience for students in the development of embedded projects, laying a solid foundation for students to further engage in embedded-related work. With the advent of the Internet of Things, the society has placed ever-increasing demands on embedded talents, and embedded development courses will inevitably face more opportunities and challenges. Our future research will be focused on the verifications on the performance of the proposed teaching methodology.

\section{Conflicts of Interest}

The author declares no conflicts of interest regarding the publication of this paper.

\section{References}

[1] Rantala V., Könnölä K., Suomi S., Isomäki M. and Lehtonen T. (2017) . Agile Embedded System Development Versus European Space Standards. International Journal of Information Systems and Social Change (IJISSC), 8(1): 1-23.

[2] Samkaria R., Singh R., Gehlot A., Yadav M.S., Kumar A., Choudhary V., Choudhury S., Pachauri R. and Aggarwal A. (2018). Embedded-Based Smart Solar Grid of $2 \times 2$ Monitoring System Using Smart Sensors. In Intelligent Communication, Control and Devices, 1777-1788.

[3] Kovshoff H., Cebula K., Tsai H.W.J. and Hastings R.P. (2017). Siblings of Children with Autism: The Siblings Embedded Systems Framework. Current Developmental Disorders Reports, 4(2): 7-45.

[4] Liu X., Wang, Z., Wu G. and Zheng Y. (2017). Monitoring and Controlling System of Maize Precision Sowing and Fertilization Based on Embedded Technology. In 2017 ASABE Annual International Meeting, American Society of Agricultural and Biological Engineers, 1.

[5] Cosío-León M.A., Ojeda-Carreño D., Nieto-Hipólito J.I. and Ibarra-Hernández J.A. (2017). The Use of Standards in Embedded Devices to Achieve End to End Semantic Interoperability on Health Systems. Computer Standards \& Interfaces.

[6] Grobelna I., Wiśniewski R., Grobelny M. and Wiśniewska M. (2017). Design and Verification of Real-life Processes with Application of Petri Nets. IEEE Transactions on Systems, Man, and Cybernetics: Systems, 47(11): 28562869.

[7] Deng L., Li D., Yao X., Cox D. and Wang H. (2018). Mobile Network Intrusion Detection for IoT System Based on Transfer Learning Algorithm. Cluster Computing, 1-16.

[8] Hirayama M. (2017). Usability Test Method for Embedded Systems Considering with Visually Impaired. In Proceedings of the 2017 International Conference on Software and e-Business, ACM, 102-106.

[9] Wan L., Skoko, J., Yu J., LeDuc P.R. and Neumann C.A. (2017). Mimicking Embedded Vasculature Structure for 3D Cancer on a Chip Approaches through Micromilling. Scientific Reports, 7(1), 16724.

[10] Rasal S.U., Gupta K., Rasal, V. and Shelar, S. (2017). Automotive Refuge Concept Using Embedded and Computing Approaches. International Journal of Advanced Research in Computer Science, 8(5).

[11] Royuela S., Duran A., Serrano M.A., Quiñones E. and Martorell X. (2017). A Functional Safety OpenMP $\$ \$^{\wedge}\{*\} \$ \$$ for Critical Real-Time Embedded Systems. In International Workshop on OpenMP, 231-245.

[12] Zhou X., Min, X., Ji Y., Gao N., Li Y., Yao X. and Xue K. (2017) August. Fabrication of High Q Factor Integrated Passive Devices Based on Embedded Fan-out Wafer Level Package. 2017 18th International Conference on Electronic Packaging Technology (ICEPT), IEEE, 601-604. 
[13] Sebastian E.J., Jose J.M., Schappacher M. and Sikora A. (2017). Seamless Test Environment for Distributed Embedded Wireless Networks. 2017 International Conference on Advances in Computing, Communications and Informatics (ICACCI), IEEE, 681-686.

[14] Deschambault O., Gherbi A. and Legare C. (2017). Efficient Implementation of the MQTT Protocol for Embedded Systems. Journal of Information Processing Systems, 13(1): 26-39. 